\title{
Model Checking with Strong Fairness *
}

\author{
Yonit Kesten $^{\dagger} \quad$ Amir Pnueli $^{\ddagger} \quad$ Li-on Raviv $^{\ddagger} \quad$ Elad Shahar ${ }^{\ddagger}$
}

August 12, 2003

\begin{abstract}
In this paper we present a coherent framework for symbolic model checking of linear-time temporal logic (LTL) properties over finite state reactive systems, taking full fairness constraints into consideration. We use the computational model of a fair discrete system (FDS) which takes into account both justice (weak fairness) and compassion (strong fairness). The approach presented here reduces the model-checking problem into the question of whether a given FDS is feasible (i.e. has at least one computation).

The contribution of the paper is twofold: On the methodological level, it presents a direct self-contained exposition of full LTL symbolic model checking without resorting to reductions to either $\mu$-calculus, CTL or automata. On the technical level, it extends previous methods by dealing with compassion at the algorithmic level instead of either adding it to the specification, or transforming compassion to justice.

Finally, we extend $\mathrm{CTL}^{*}$ with past operators, and show that the basic symbolic feasibility algorithm presented here, can be used to model check an arbitrary CTL* formula over an FDS with full fairness constraints.
\end{abstract}

\section{Introduction}

Two kinds of temporal logics have been proposed over the years for specifying the properties of reactive systems: the linear time logic LTL [GPSS80] and the branching time variant CTL [CE81]. Also two methods for the formal verification of the temporal properties of reactive systems have been developed: the deductive approach based on interactive theorem proving, and the fully automatic algorithmic approach, widely known as model checking. Tracing the evolution of these ideas, we find that the deductive approach adopted LTL as its main vehicle for specification, while the modelchecking approach used CTL as the specification language [CE81], [QS82].

This is more than a historical coincidence or a matter of personal preference. The main advantage of CTL for model checking is that it is state-based and, therefore, the process of verification can be performed by straightforward labeling of the existing states in the discrete structure, leading to no further expansion or unwinding of the structure. In contrast, LTL is path-based and, since many paths can pass through a single state, labeling a structure by the LTL sub-formulas it satisfies necessarily requires splitting the state into several copies. This is the reason why the development of model-checking algorithms for LTL always lagged several years behind their first introduction for the CTL logic.

${ }^{*}$ This research was supported in part by an infra-structure grant from the Israeli Ministry of Science and Art, a grant from the U.S.-Israel Binational Science Foundation, and a gift from Intel.

${ }^{\dagger}$ Dept. of Communication Systems Engineering, Ben Gurion University, ykesten@bgumail.bgu . ac.il — Contact author

${ }^{\ddagger}$ Weizmann Institute of Science, $\{$ amir, elad\}@wisdom.weizmann.ac.il 
The first model-checking algorithms were based on the enumerative approach, constructing an explicit representation of all reachable states of the considered system [CE81], and were developed for the branching-time temporal logic CTL. The LTL version of these algorithms was developed in [LP85] for the future fragment of propositional LTL (PTL), and extended in [LPZ85] to the full PTL. The basic fixed-point computation algorithm for the identification of fair computations presented in [LP85], was developed independently in [EL87] for FCTL (fair CTL).

Observing that upgrading from justice to full fairness (i.e., adding compassion) is reflected in the automata view of verification as an upgrade from a Büchi to a Streett automaton, we can view the algorithms presented in [EL87] and [LP85] as algorithms for checking the emptiness of Streett automata [VW86]. An improved algorithm solving the related problem of emptiness of Streett automata, was later presented in [HT96]. The development of the impressively efficient symbolic verification methods and their application to $\mathrm{CTL}\left[\mathrm{BCM}^{+} 92\right]$ raised the question whether a similar approach can be applied to PTL. The first satisfactory answer to this question is given in $\left[\mathrm{BCM}^{+} 92\right]$, by showing a reduction of PTL (future fragment) model checking into $\mu$-calculus model checking. A similar transformation from LTL to CTL model checking, is presented in [CGH97]. The advantage of this approach is that, following a preliminary transformation of the PTL formula and the given system, the algorithm proceeds by using available and efficient CTL symbolic model checkers such as SMV.

A certain weakness of all the available symbolic model checkers is that, in their representation of fairness, they only consider the concept of justice (weak fairness). As suggested by many researchers, another important fairness requirement is that of compassion (strong fairness) (e.g., [GPSS80], [LPS81], [Fra86]). This type of fairness is particularly useful in the analysis of systems that use semaphores, synchronous communication, and other special coordination primitives. A partial answer to this criticism is that, since compassion can be expressed in LTL (but not in CTL), once we developed a model-checking method for LTL, we can always add the compassion requirements as an antecedent to the property we wish to verify. A similar answer is standardly given for symbolic model checkers that use the $\mu$-calculus as their specification language, because compassion can also be expressed as a $\mu$-calculus formula [SdRG89]. The only question remaining is how practical this is.

In this paper we present an approach to the symbolic model checking of PTL formulas, which takes into account full fairness, including both justice and compassion. The approach is selfcontained and does not depend on a reduction to either $\mu$-calculus or CTL model checking (as in $\left[\mathrm{BCM}^{+} 92\right]$ and [CGH97], respectively) or to automata. The main advantage of such a self-contained approach is that the end users no longer need to deal with two different kinds of logics.

The treatment of the PTL component is essentially that of a symbolic construction of a tableau by assigning a new auxiliary variable to each temporal sub-formula of the property we wish to verify. In that, our approach resembles very much the reduction method used in [BCM ${ }^{+}$92, CGH97] which, in turn, is an extension of the statification method used in [MP91a] and [MP95] to deal with the past fragment of LTL. The model-checking problem is then reduced into the question of feasibility of an FDS. The symbolic feasibility algorithm, similar to the enumerative algorithm of [LP85], identifies all computations satisfying a given set of fairness constraints. This involves the identification of all fair strongly connected components (SCC). However, while the enumerative algorithm identifies each SCC separately, the BDD-based symbolic algorithm is more efficient, identifying all states participating in some fair SCC simultaneously. Our symbolic algorithm can be viewed as a straightforward implementation of the nested fixed-point characterization of EmersonLei for fully fair computations [EL86], as opposed to the CTL model checkers which consider only the weak-fairness part of this characterization. 
Other works related to the approach developed here are presented in [HTKB92] [HKSV97], where BDD-based symbolic algorithms for bad cycle detection are presented. They are based on the fixed-point characterization of Emerson-Lei. These algorithm solves the problem of finding all those cycles within the computation graph, which satisfy a given set of weak fairness constraints. [HKSV97] gives heuristics which improves the performance of the Emerson-Lei algorithm. We use the same heuristics in our algorithm, while dealing with both types of fairness constraints. [HTKB92] [HKSV97] can deal with strong fairness by reduction to weak fairness. According to the automata-theoretic view, [HKSV97] presents a symbolic algorithm for the problem of emptiness of Büchi automata, while the algorithms presented here provide a symbolic solution to the emptiness problem of Streett automata.

An algorithm for dealing with compassion at the algorithmic level is presented in [Kur95], however it is enumerative whereas our work provides a symbolic solution.

In [EL87], Emerson and Lei observed that the problem of $\mathrm{CTL}^{*}$ model checking of finite state systems can be resolved by recursive calls to an LTL model-checking algorithm. Taking a similar approach, we augment $\mathrm{CTL}^{*}$ with past operators and show that the symbolic feasibility algorithm presented here, can be used to model check an arbitrary CTL* formula over a finite state FDS $\mathcal{D}$, taking the full fairness constraints of $\mathcal{D}$ into consideration.

The rest of the paper is organized as follows. In section 2 we present the computational model of fair discrete systems (FDS). In section 3 we present PTL, the propositional fragment of linear temporal logic, including the past operators. Next, in section 4 we discuss the construction of a tester for a PTL formula $\varphi$, which is an FDS characterizing all the sequences which satisfy $\varphi$. Having transformed the model-checking problem into the feasibility problem of an FDS, we present the symbolic feasibility algorithm in section 5, followed by an algorithm for extracting a witness (a counter example) in section 6 . In section 7 we augment $\mathrm{CTL}^{*}$ with past operators and use the feasibility algorithm to model check an arbitrary $\mathrm{CTL}^{*}$ formulas over a finite state FDS. We conclude in section 8 with some experimental results, comparing the different methods used to deal with compassion requirements.

A (partial) conference version of this paper appeared in [KPR98].

\section{Fair Discrete Systems}

As a computational model for reactive systems, we take the model of fair discrete system (FDS). The computational model is used for modeling both the verified system and the temporal properties. The FDS model replaces the earlier model of fair transition system (FTS) presented in [MP91b] and [MP95]. The main difference between these two models is in the representation of fairness constraints. The advantage of the new representation is that it enables a unified representation of fairness constraints arising from both the system being verified, and the temporal property.

An FDS $\mathcal{D}:\langle V, \Theta, \rho, \mathcal{J}, \mathcal{C}\rangle$ consists of the following components:

- $V=\left\{u_{1}, \ldots, u_{n}\right\}$ : A finite set of typed state variables ranging over finite domains. We define a state $s$ to be a type-consistent interpretation of $V$, assigning to each variable $u \in V$ a value $s[u]$ in its domain. We denote by $\Sigma$ the set of all states.

- $\Theta$ : The initial condition. This is an assertion characterizing all the initial states of the $\mathcal{D}$. A state is called initial if it satisfies $\Theta$.

- $\rho$ : A transition relation. This is an assertion $\rho\left(V, V^{\prime}\right)$, relating a state $s \in \Sigma$ to its $\mathcal{D}$-successor $s^{\prime} \in \Sigma$ by referring to both unprimed and primed versions of the state variables. The transition 
relation $\rho\left(V, V^{\prime}\right)$ identifies state $s^{\prime}$ as a $\mathcal{D}$-successor of state $s$ if $\left\langle s, s^{\prime}\right\rangle \models \rho\left(V, V^{\prime}\right)$, where $\left\langle s, s^{\prime}\right\rangle$ is the joint interpretation which interprets $x \in V$ as $s[x]$, and $x^{\prime}$ as $s^{\prime}[x]$.

- $\mathcal{J}=\left\{J_{1}, \ldots, J_{k}\right\}$ : A set of assertions expressing the justice (weak fairness) requirements. Intentionally, the justice requirement $J \in \mathcal{J}$ stipulates that every computation contains infinitely many $J$-states (states satisfying $J$ ).

- $\mathcal{C}=\left\{\left\langle p_{1}, q_{1}\right\rangle, \ldots\left\langle p_{n}, q_{n}\right\rangle\right\}$ : A set of assertions expressing the compassion (strong fairness) requirements . Intentionally, the compassion requirement $\langle p, q\rangle \in \mathcal{C}$ stipulates that every computation containing infinitely many $p$-states also contains infinitely many $q$-states.

Let $\sigma: s_{0}, s_{1}, \ldots$, be a sequence of states, $\varphi$ be an assertion, and $j \geq 0$ be a natural number. We say that $j$ is a $\varphi$-position of $\sigma$ if $s_{j}$ is a $\varphi$-state, namely, if $\varphi$ holds on $s$. Let $\mathcal{D}$ be an FDs for which the above components have been identified. We define a run of $\mathcal{D}$ to be an infinite sequence of states $\sigma: s_{0}, s_{1}, \ldots$, satisfying the requirements:

- Initiality: $\quad s_{0}$ is initial, i.e., $s_{0} \models \Theta$.

- Consecution: For each $j=0,1, \ldots$, the state $s_{j+1}$ is a $\mathcal{D}$-successor of the state $s_{j}$.

A run of $\mathcal{D}$ is called a computation if it satisfies the following fairness requirements:

- Justice: $\quad$ For each $J \in \mathcal{J}, \sigma$ contains infinitely many $J$-positions

- Compassion: For each $\langle p, q\rangle \in \mathcal{C}$, if $\sigma$ contains infinitely many $p$-positions, it also contain infinitely many $q$-positions.

We denote by $\operatorname{Comp}(\mathcal{D})$ the set of all computations of $\mathcal{D}$.

A state $s$ is said to be $\mathcal{D}$-reachable if it participates in a run of $\mathcal{D}$. We say that a state is $\mathcal{D}$-feasible if it participates in some computation of $\mathcal{D}$. An FDS $\mathcal{D}$ is feasible if $\mathcal{D}$ has at least one computation. We say that an FDS $\mathcal{D}$ is viable if every $\mathcal{D}$-reachable state is $\mathcal{D}$-feasible. Note that the FDS model does not guarantee viability.

\section{Parallel Composition of FDS's}

Fair discrete systems can be composed in parallel. Let $\mathcal{D}_{i}=\left\langle V_{i}, \Theta_{i}, \rho_{i}, \mathcal{J}_{i}, \mathcal{C}_{i}\right\rangle, i \in\{1,2\}$, be two fair discrete systems. Two versions of parallel composition are used.

Asynchronous composition is used to assemble an asynchronous system from its components. We define the asynchronous parallel composition of two FDS's to be

$$
\langle V, \Theta, \rho, \mathcal{J}, \mathcal{C}\rangle=\left\langle V_{1}, \Theta_{1}, \rho_{1}, \mathcal{J}_{1}, \mathcal{C}_{1}\right\rangle \|\left\langle V_{2}, \Theta_{2}, \rho_{2}, \mathcal{J}_{2}, \mathcal{C}_{2}\right\rangle
$$

where,

$$
\begin{aligned}
& V=V_{1} \cup V_{2} \quad \Theta=\Theta_{1} \wedge \Theta_{2} \quad \rho=\rho_{1} \vee \rho_{2} \\
& \mathcal{J}=\mathcal{J}_{1} \cup \mathcal{J}_{2} \quad \mathcal{C}=\mathcal{C}_{1} \cup \mathcal{C}_{2}
\end{aligned}
$$

We can view the asynchronous execution of $\mathcal{D}$ as the interleaved execution of $\mathcal{D}_{1}$ and $\mathcal{D}_{2}$.

Synchronous composition is used in some cases, to assemble a system from its components (in particular when considering hardware designs which are naturally synchronous). However, our primary use of synchronous composition is for combining a system with a tester $T_{\varphi}$ for an LTL formula $\varphi$ (see section 4). We define the synchronous parallel composition of two FDs's to be

$$
\langle V, \Theta, \rho, \mathcal{J}, \mathcal{C}\rangle=\left\langle V_{1}, \Theta_{1}, \rho_{1}, \mathcal{J}_{1}, \mathcal{C}_{1}\right\rangle \|\left|V_{2}, \Theta_{2}, \rho_{2}, \mathcal{J}_{2}, \mathcal{C}_{2}\right\rangle
$$


where,

$$
\begin{aligned}
& V=V_{1} \cup V_{2} \quad \Theta=\Theta_{1} \wedge \Theta_{2} \quad \rho=\rho_{1} \wedge \rho_{2} \\
& \mathcal{J}=\mathcal{J}_{1} \cup \mathcal{J}_{2} \quad \mathcal{C}=\mathcal{C}_{1} \cup \mathcal{C}_{2}
\end{aligned}
$$

The synchronous parallel composition of systems $\mathcal{D}_{1}$ and $\mathcal{D}_{2}$ is a new system $\mathcal{D}$, each of whose basic actions consists of the joint execution of an action of $\mathcal{D}_{1}$ and an action of $\mathcal{D}_{2}$. We can view the execution of $\mathcal{D}$ as the joint execution of $\mathcal{D}_{1}$ and $\mathcal{D}_{2}$.

\section{$3 \quad$ Linear Temporal Logic}

As a requirement specification language for reactive systems we take the propositional fragment of linear temporal logic (PTL) [MP91b].

Let $\mathcal{P}$ be a finite set of propositions. A state formula is constructed out of propositions and the boolean operators $\neg$ and $\vee$. A temporal formula is constructed out of state formulas to which we apply the boolean operators and the following basic temporal operators:

$$
\begin{array}{ll}
\bigcirc-\text { Next } & \ominus-\text { Previous } \\
\mathcal{U} \text { - Until } & \mathcal{S} \text { - Since }
\end{array}
$$

We refer to the set of variables that occur in a formula $p$ as the vocabulary of $p$. A model for a temporal formula $p$ is an infinite sequence of states $\sigma: s_{0}, s_{1}, \ldots$, where each state $s_{j}$ provides an interpretation for the vocabulary of $p$.

Given a model $\sigma$, as above, we present an inductive definition for the notion of a temporal formula $p$ holding at a position $j \geq 0$ in $\sigma$, denoted by $(\sigma, j) \models p$.

- For a state formula $p$,

$$
(\sigma, j) \models p \quad \Leftrightarrow \quad s_{j} \models p
$$

That is, we evaluate $p$ locally, using the interpretation given by $s_{j}$.

- $(\sigma, j) \models \neg p \quad \Leftrightarrow \quad(\sigma, j) \not \models p$

- $(\sigma, j) \models p \vee q \quad \Leftrightarrow \quad(\sigma, j) \models p$ or $(\sigma, j) \models q$

- $(\sigma, j) \models \bigcirc p \quad \Leftrightarrow \quad(\sigma, j+1) \models p$

- $(\sigma, j) \models p \mathcal{U} q \quad \Leftrightarrow \quad$ for some $k \geq j,(\sigma, k) \models q$,

and for every $i$ such that $j \leq i<k,(\sigma, i) \models p$

- $(\sigma, j) \models \ominus p \quad \Leftrightarrow \quad j>0$ and $(\sigma, j-1) \models p$

- $(\sigma, j) \models p \mathcal{S} q \quad \Leftrightarrow \quad$ for some $k \leq j,(\sigma, k) \models q$,

and for every $i$ such that $k<i \leq j,(\sigma, i) \models p$

If $(\sigma, 0) \models p$, we say that $p$ holds on $\sigma$, and denote it by $\sigma \models p$. A formula $p$ is called satisfiable if it holds on some model. A formula is called temporally valid if it holds on all models.

Given an FDS $\mathcal{D}$, we can restrict our attention to the set of models which correspond to computations of $\mathcal{D}$, i.e., $\operatorname{Comp}(\mathcal{D})$. This leads to the notion of $\mathcal{D}$-validity, by which a temporal formula $p$ is $\mathcal{D}$-valid (valid over FDS $\mathcal{D}$ ) if it holds over all the computations of $\mathcal{D}$. Obviously, any formula that is (generally) valid is also $\mathcal{D}$-valid for any FDS $\mathcal{D}$. In a similar way, we obtain the notion of $\mathcal{D}$-satisfiability.

Additional temporal operators may be defined as follows:

$$
\begin{aligned}
& \diamond p=\mathrm{T} \mathcal{U} p \quad-\text { Eventually } p \\
& \square p \quad=\neg \diamond \neg p \quad-\text { Always, henceforth } p \\
& p \mathcal{W} q=\neg((\neg q) \mathcal{U}(\neg p \wedge \neg q) \quad-\quad \text { Waiting-for, unless, weak until }
\end{aligned}
$$




\section{Construction of Testers for LTL Formulas}

In this section, we present the construction of a tester for a PTL formula $\varphi$, which is an FDS $T_{\varphi}$ characterizing all the sequences which satisfy $\varphi$. Without loss of generality, assume that the only temporal operators occurring in $\varphi$ are $\bigcirc, \mathcal{U}, \Theta$ and $\mathcal{S}$.

For a formula $\psi$, we write $\psi \in \varphi$ to denote that $\psi$ is a sub-formula of (possibly equal to) $\varphi$. Formula $\psi$ is called principally temporal if its main operator is a temporal operator. The FDS $T_{\varphi}$ is given by

$$
T_{\varphi}:\left\langle V_{\varphi}, \Theta_{\varphi}, \rho_{\varphi}, \mathcal{J}_{\varphi}, \mathcal{C}_{\varphi}\right\rangle
$$

where the components are specified as follows:

\section{System Variables}

The system variables of $T_{\varphi}$ consist of the vocabulary of $\varphi$ plus a set of auxiliary boolean variables

$$
X_{\varphi}: \quad\left\{x_{p} \mid p \in \varphi \text { a principally temporal sub-formula of } \varphi\right\}
$$

which includes an auxiliary variable $x_{p}$ for every $p$, a principally temporal sub-formula of $\varphi$. The auxiliary variable $x_{p}$ is intended to be true in a state of a computation iff the temporal formula $p$ holds at that state.

We define a mapping $\chi$ which maps every sub-formula of $\varphi$ into an assertion over $V_{\varphi}$.

$$
\chi(\psi)= \begin{cases}\psi & \text { for } \psi \text { a state formula } \\ \neg \chi(p) & \text { for } \psi=\neg p \\ \chi(p) \vee \chi(q) & \text { for } \psi=p \vee q \\ x_{\psi} & \text { for } \psi \text { a principally temporal formula }\end{cases}
$$

The mapping $\chi$ distributes over all boolean operators. When applied to a state formula it yields the formula itself. When applied to a principally temporal sub-formula $p$ it yields $x_{p}$.

\section{Initial Condition}

The initial condition of $T_{\varphi}$ is given by

where

$$
\Theta_{\varphi} \quad=\operatorname{past}-\operatorname{init}(\varphi)
$$

$$
\operatorname{past-init}(\varphi)=\bigwedge_{\Theta p \in \varphi} \neg x_{\Theta p} \wedge \bigwedge_{p \mathcal{S} q \in \varphi}\left(x_{p \mathcal{S}_{q}} \leftrightarrow \chi(q)\right)
$$

Thus, the initial condition requires that all auxiliary variables encoding "Previous" formulas are initially false. This corresponds to the observation that all formulas of the form $\ominus p$ are false at the first state of any sequence. In addition, past-init $(\varphi)$ requires that the truth value of $x_{p \mathcal{S}_{q}}$ equals the truth value of $\chi(q)$, corresponding to the observation that the only way to satisfy the formula $p \mathcal{S} q$ at the first state of a sequence is by satisfying $q$.

Note that, unlike the definition of testers presented in [KPR98, KP00], the assertion $\chi(\varphi)$ is not a conjunct of $\Theta_{\varphi}$. Namely, the initial condition of a tester $T_{\varphi}$ does not assert $\chi(\varphi)$. This will permit the use of algorithm FEASIBLE presented in Section 5, for model checking both LTL and $\mathrm{CTL}^{*}$ properties, as discussed in section 7 . 


\section{Transition Relation}

The transition relation of $T_{\varphi}$ is given by

$$
\rho_{\varphi}: \quad\left(\begin{array}{ccc}
\bigwedge_{\ominus p \in \varphi}\left(x_{\ominus p}^{\prime} \leftrightarrow \chi(p)\right) & \wedge \bigwedge_{p \mathcal{S} q \in \varphi}\left(x_{p \mathcal{S}_{q}}^{\prime} \leftrightarrow\left(\chi^{\prime}(q) \vee\left(\chi^{\prime}(p) \wedge x_{p \mathcal{S}_{q}}\right)\right)\right) \\
\wedge \bigwedge_{\bigcirc p \in \varphi}\left(x_{\bigcirc p} \leftrightarrow \chi^{\prime}(p)\right) & \left.\wedge \bigwedge_{p \mathcal{U}_{q \in \varphi} \in x_{p \mathcal{U} q}} \leftrightarrow\left(\chi(q) \vee\left(\chi(p) \wedge x_{p \mathcal{U}_{q}}^{\prime}\right)\right)\right)
\end{array}\right)
$$

Note that we use the form $x_{\psi}$ when we know that $\psi$ is principally temporal and the form $\chi(\psi)$ in all other cases. The expression $\chi^{\prime}(\psi)$ denotes the primed version of $\chi(\psi)$. The conjuncts of the transition relation corresponding to the Since and the Until operators are based on the following expansion formulas:

$$
p \mathcal{S} q \Longleftrightarrow q \vee(p \wedge \ominus(p \mathcal{S} q)) \quad p \mathcal{U} q \quad \Longleftrightarrow q \vee(p \wedge \bigcirc(p \mathcal{U} q))
$$

where $\Longleftrightarrow$ denotes congruence, namely $(a \Longleftrightarrow b)=\square(a \leftrightarrow b)$.

\section{Fairness Requirements}

For each formula $p \mathcal{U} q \in \varphi$ which has a positive occurrence in $\varphi$ (i.e., an occurrence under an even number of negations), we include in $\mathcal{J}$ the disjunction

$$
\chi(q) \vee \neg x_{p} \mathcal{U}_{q}
$$

This justice requirement ensures that the sequence contains infinitely many states at which $\chi(q)$ is true, or infinitely many states at which $x_{p \mathcal{u}_{q}}$ is false. The compassion set of $T_{\varphi}$ is always empty.

\section{Correctness of the Construction}

For a set of variables $U$, we say that sequence $\widetilde{\sigma}$ is a $U$-variant of sequence $\sigma$ if $\sigma$ and $\tilde{\sigma}$ agree on the interpretation of all variables, except possibly the variables in $U$.

The following claim states that the construction of the tester $T_{\varphi}$ correctly captures the set of sequences satisfying the formula $\varphi$.

Claim 1 A state sequence $\sigma=s_{0}, \ldots$ satisfies the temporal formula $\varphi$ iff $\sigma$ is an $X_{\varphi}$-variant of a computation $\widetilde{\sigma}=\widetilde{s_{0}}, \ldots$ of $T_{\varphi}$, and $\widetilde{s_{0}}=\chi(\varphi)$.

\section{Checking for Feasibility}

In this section we present a symbolic algorithm for computing the set of $\mathcal{D}$-feasible states. The symbolic algorithm presented here is inspired by the full state-enumeration algorithm originally presented in [LP85] and [EL87] (for full explanations and proofs see [Lic91] and [MP95]). The enumerative algorithm was designed for LTL model checking, and was concerned with checking feasibility of an FDS. Since we want to use the same basic algorithm for both LTL and CTL* model checking, our basic symbolic algorithm computes the set of $\mathcal{D}$-feasible states, from which the feasibility of $\mathcal{D}$ is trivially obtained. The enumerative algorithm constructs a state-transition graph $G_{\mathcal{D}}$ for $\mathcal{D}$. This is a directed graph whose nodes are all the $\mathcal{D}$-reachable states, and whose edges connect node $s$ to node $s^{\prime}$ iff $s^{\prime}$ is a $\mathcal{D}$-successor of $s$. If system $\mathcal{D}$ has a computation it corresponds 
to an infinite path in the graph $G_{\mathcal{D}}$ which starts at a $\mathcal{D}$-initial state. We refer to such paths as initialized paths.

Subgraphs of $G_{\mathcal{D}}$ can be specified by identifying a subset $S \subseteq G_{\mathcal{D}}$ of the nodes of $G_{\mathcal{D}}$. It is implied that as the edges of the subgraph we take all the original $G_{\mathcal{D}}$-edges connecting nodes (states) of $S$. A subgraph $S$ is called just if it contains a $J$-state for every justice requirement $J \in \mathcal{J}$. The subgraph $S$ is called compassionate if, for every compassion requirement $(p, q) \in \mathcal{C}, S$ contains a $q$-state, or $S$ does not contain any $p$-state. A subgraph is singular if it is composed of a single state which is not connected to itself. A subgraph $S$ is fair if it is a non-singular strongly connected subgraph (SCS) which is both just and compassionate.

For $\pi$, an infinite initialized path in $G_{\mathcal{D}}$, we denote by $\operatorname{Inf}(\pi)$ the set of states which appear infinitely many times in $\pi$. The following claim, which is proved in [Lic91], connects computations of $\mathcal{D}$ with fair subgraphs of $G_{\mathcal{D}}$.

Claim 2 The infinite initialized path $\pi$ is a computation of $\mathcal{D}$ iff $\operatorname{Inf}(\pi)$ is a fair subgraph of $G_{\mathcal{D}}$.

\section{The Symbolic algorithm}

The symbolic algorithm, aimed at exploiting the data structure of OBDD's, is presented in a general set notation. Let $\Sigma$ denote the set of all states of an FDS $\mathcal{D}$. A predicate over $\Sigma$ is any subset $U \subseteq \Sigma$. A (binary) relation over $\Sigma$ is any set of pairs $R \subseteq \Sigma \times \Sigma$. Since both predicates and relations are sets, we can freely apply the set-operations of union, intersection, and complementation to these objects. In addition, we define two operations of composition of predicates and relations. For a predicate $U$ and relation $R$, we define the operations of pre- and post-composition as follows:

$$
\begin{aligned}
& R \circ U=\left\{s \in \Sigma \mid\left(s, s^{\prime}\right) \in R \text { for some } s^{\prime} \in U\right\} \\
& U \circ R=\left\{s \in \Sigma \mid\left(s_{0}, s\right) \in R \text { for some } s_{0} \in U\right\}
\end{aligned}
$$

If we view $R$ as a transition relation, then $R \circ U$ is the set of all $R$-predecessors of $U$-states, and $U \circ R$ is the set of all $R$-successors of $U$-states. To capture the set of all states that can reach a $U$-state in a finite number of $R$-steps (including zero), we define

$$
R^{*} \circ U=U \cup R \circ U \cup R \circ(R \circ U) \cup R \circ(R \circ(R \circ U)) \cup \cdots .
$$

It is easy to see that $R^{*} \circ U$ converges after a finite number of steps. In a similar way, we define

$$
U \circ R^{*}=U \cup U \circ R \cup(U \circ R) \circ R \cup((U \circ R) \circ R) \circ R \cup \cdots,
$$

which captures the set of all states reachable in a finite number of $R$-steps from a $U$-state. For predicates $U$ and $W$, we define the relation $U \times W$ as

$$
U \times W=\left\{\left(s_{1}, s_{2}\right) \in \Sigma^{2} \mid s_{1} \in U, s_{2} \in W\right\} .
$$

Let $\mathcal{D}:\langle V, \Theta, \rho, \mathcal{J}, \mathcal{C}\rangle$ be an FDS. For an assertion $\varphi$ over $V$, the system variables of $\mathcal{D}$, we denote by $\|\varphi\|$ the predicate consisting of all states satisfying $\varphi$. Similarly, for an assertion $\rho$ over $\left(V, V^{\prime}\right)$, we denote by $\|\rho\|$ the relation consisting of all state pairs $\left\langle s, s^{\prime}\right\rangle$ satisfying $\rho$.

Algorithm FEASIBLE presented in Fig. 1, denotes by $R$ the transition relation implied by $\rho$. The predicate variable new represents a set of states. We use the shorthand notation

$$
R \cap n e w \quad \stackrel{\text { def }}{=} R \cap(n e w \times \Sigma) .
$$


Algorithm feasible $(\mathcal{D})$ : predicate - Compute the set of $\mathcal{D}$-feasible states

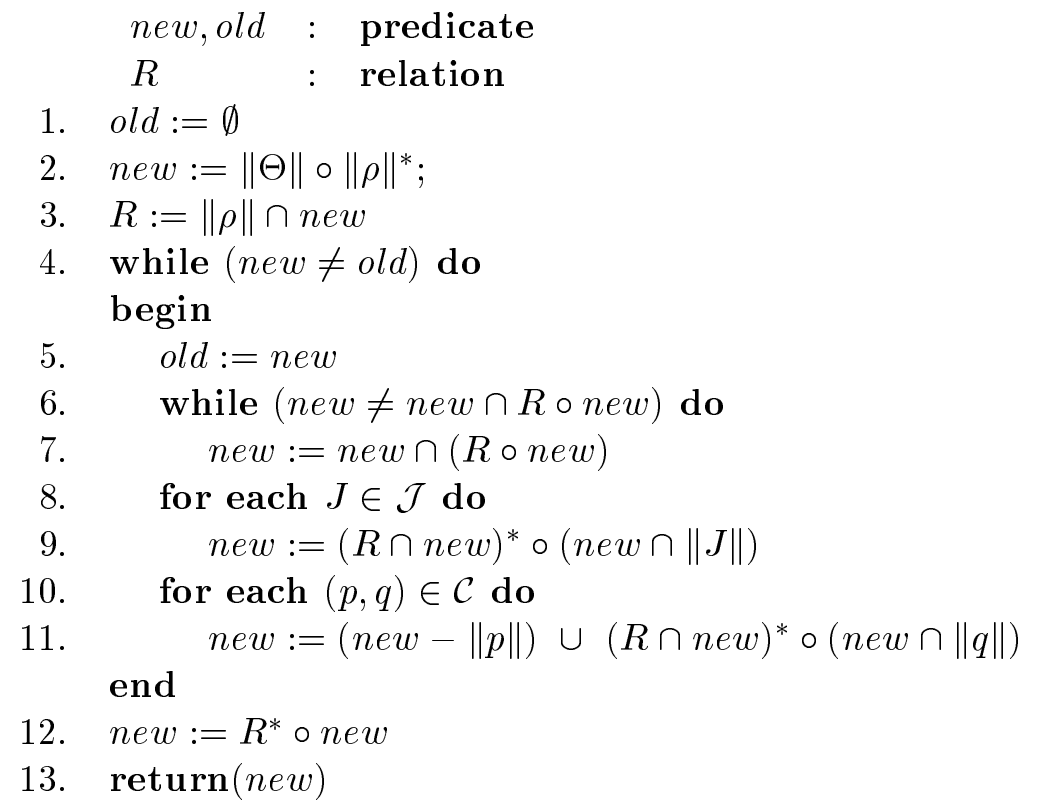

Figure 1: Algorithm FEASIBLE

Algorithm FEASIBLE consists of a main loop which converges when the values of the predicate variable new coincide on two successive visits to line 4 . Prior to entry to the main loop we compute in new the universal set of all reachable states in $\mathcal{D}$ and place in $R$ the transition relation implied by $\rho$, restricted to pairs $\left(s_{1}, s_{2}\right)$ where $s_{1}$ is a reachable state in $\mathcal{D}$.

The main loop (lines $4-11$ ), contains three inner loops. The inner loop at lines $6-7$, successively removes from new all states which do not have a successor in new. The loop at lines 8-9 removes from new all states which are not $R^{*}$-predecessors of some $J$-state, for all justice requirements $J \in \mathcal{J}$. The term $R \cap$ new restricts $R$ to pairs $\left(s_{1}, s_{2}\right)$ where $s_{1}$ is currently in new. This is done to avoid regeneration of states which have already been eliminated. The loop at lines 10-11, removes from new all $p$-states which are not $R^{*}$-predecessors of some $q$-state for some $(p, q) \in \mathcal{C}$. The term $R \cap$ new restricts $R$ again to pairs $\left(s_{1}, s_{2}\right)$ where $s_{1}$ is currently in new.

Finally, line 12 augments new with all its $R^{*}$-predecessors.

\section{Correctness of the FEAsiBle Algorithm}

The following sequence of claims establishes the correctness of the algorithm.

Claim 3 (Termination) Algorithm FEASIBLE terminates.

Proof: Let us denote by new $w_{i}^{4}$ the value of variable new on the $i$ 'th visit $(i=1,2, \ldots)$ to line 4 of the algorithm. Since all assignments to variable new within the main loop, are of the form $n e w:=E$ where $E \subseteq n e w$, it is not difficult to see that $n e w_{2}^{4} \subseteq n e w_{1}^{4}$. From this, it can be established by induction on $i$ that $n e w_{i+1}^{4} \subseteq n e w_{i}^{4}$, for every $i=1,2 \ldots$. It follows that the sequence $\mid$ new $w_{1}^{4}|\geq|$ new $w_{2}^{4}|\geq|$ new $w_{3}^{4} \mid \cdots$, is a non-increasing sequence of natural numbers which 
must eventually stabilize. At the point of stabilization, we have that $n e w_{i+1}^{4}=n e w_{i}^{4}$, implying termination of the algorithm.

Let $\mathcal{D}:\langle V, \Theta, \rho, \mathcal{J}, \mathcal{C}\rangle$ be an FDS, $s$ be a $\mathcal{D}$-reachable state and $U$ be the set of states resulting from the application of algorithm FEASIBLE over $\mathcal{D}$.

Claim 4 (Completeness) If $s$ is $\mathcal{D}$-feasible then $s \in U$.

Proof: Assume that $s$ is $\mathcal{D}$-feasible. Then by definition, $s$ is on an initialized fair path $\pi$ in $\mathcal{D}$. From Claim 2, $\operatorname{Inf}(\pi)$ is a fair subgraph $S \subseteq G_{\mathcal{D}}$. Namely, $S$ is a non-singular strongly-connected subgraph (SCS) which contains a $J$-state for every $J \in \mathcal{J}$, and such that, for every $(p, q) \in \mathcal{C}, S$ contains a $q$-state or contains no $p$-state. Let

$$
\widetilde{S}: \quad S \cup\left\{s^{\prime} \mid s^{\prime} \text { is on an initialized path to a state in } S\right\}
$$

Obviously $s \in \widetilde{S}$. Following the operations performed by algorithm FEASIBLE, we can show that $S$ is contained in the set new at all locations beyond the first visit to line 4 . This is because any removal of states from new which is carried out in lines 7, 9, and 11, cannot remove any state of $S$. Consequently, $S$ must remain throughout the process and will be contained in $U$. Finally, when line 12 is executed, the set new is augmented from $S$ to $\widetilde{S}$, implying that $s \in U$.

Claim 5 (Soundness) If $s \in U$ then $s$ is $\mathcal{D}$-feasible.

Proof: Assume that $s \in U$. Let $S \subseteq U$ be the set of states in $U$, reachable from $s$ by a $U$-path. Since $s \in U, s$ is $\mathcal{D}$-reachable. For every $J \in \mathcal{J}, s$ can reach a $J$-state by a path fully contained within $S$. For every $(p, q) \in \mathcal{C}$, either $s$ is not a $p$-state, or $s$ can reach a $q$-state by an $S$-path.

Let us decompose $S$ into maximal strongly-connected subgraphs. At least one subgraph $S_{t}$ is terminal in this decomposition, in the sense that every $S$-edge exiting an $S_{t}$-state also leads to an $S_{t}$-state. We argue that $S_{t}$ is fair. By definition, it is strongly connected. It cannot be singular, because it would consist of a single state that would have been removed on the last execution of the loop at lines 6-7. Let $r$ be an arbitrary state within $S_{t}$. For every $J \in \mathcal{J}, r$ can reach some $J$-state $\tilde{r} \in U$ by an $S$-path. Since $S_{t}$ is terminal within $S$, this path must be fully contained within $S_{t}$ and, therefore, $\widetilde{r} \in S_{t}$. In a similar way, we can show that $S_{t}$ satisfies all the compassion requirements. We can conclude that $s$ is on an initialized path to a fair subgraph, which establishes that $s$ is $\mathcal{D}$-feasible.

The Claims Completeness and Soundness lead to the following conclusion:

Corollary $6 s$ is $\mathcal{D}$-feasible iff $s \in U$.

Corollary $7 \mathcal{D}$ is feasible iff $U \cap\|\Theta\| \neq \emptyset$.

Proof: A direct result of Corollary 6 and the definition of feasibility. 


\section{Algorithm FEASIBLE for CTL* Versus LTL}

Algorithm FEASIBLE presented in Fig. 1 is designed for model-checking both LTL and CTL* properties over an FDS. In [KPR98], we present a similar algorithm designed for model-checking LTL properties only. An algorithm for LTL model checking, needs to assert the existence (non-existence) of a reachable fair SCS in some FDS $\mathcal{D}$. The original enumerative algorithms of [EL87] and [LP85] were based on recursive exploration of strongly connected subgraphs, ensuring closure under both successors and predecessors. As the work in [KPR98] shows, it is possible to relax the requirement of bi-directional closure into either closure under predecessors and search for terminal SCS components, or, alternatively, closure under successors and search for initial sCs components. This idea, which may be worth exploring even in the enumerative case, has been explored extensively in the context of symbolic model checking (see [RBS00]).

In this paper however, algorithm FEASIBLE is designed for model-checking both LTL and CTL* properties, as discussed in section 7 . In the case of $\mathrm{CTL}^{*}$, we need to evaluate the set of feasible states in $\mathcal{D}$, namely the set of all states on some reachable fair SCS, or on a finite initialized path to a reachable fair SCS. In this case, we can no longer choose arbitrarily between forward and backward closure evaluation. The only appropriate choice is backward closure, which guarantees that all terminal SCS are fair. This is the version of algorithm FEASIBLE presented in Fig. 1.

\section{Model Checking LTL Properties}

Using algorithm FEASIBLE, we can now model check an LTL property $\varphi$ over an FDS $\mathcal{D}$ as follows. To model check the $\mathcal{D}$-validity $\mathcal{D} \models \varphi$,

- Define the tester $T_{\neg \varphi}$.

- Define the synchronous parallel composition $\mathcal{A}: \mathcal{D} \| T_{\neg \varphi}$. Let $\Theta_{\mathcal{A}}$ be the initial condition of the FDS $\mathcal{A}$.

- Evaluate $\operatorname{FEAsible}\left(\mathcal{D} \| T_{\neg \varphi}\right) \cap\left\|\Theta_{\mathcal{A}} \wedge \chi(\neg \varphi)\right\|$.

The verification is based on the following Claim.

Claim $8 \mathcal{D} \models \varphi \quad$ iff $\quad \operatorname{FEASIBLE}\left(\mathcal{D} \| T_{\neg \varphi}\right) \cap\left\|\Theta_{\mathcal{A}} \wedge \chi(\neg \varphi)\right\|=\emptyset$

The proof of equivalent claims can be found in [VW86], [LP85].

\section{$6 \quad$ Extracting a Witness}

To use formal verification as an effective debugging tool in the context of verification of finite-state reactive systems checked against temporal properties, a most useful information is a computation of the system which violates the requirement, to which we refer as a witness or a counter-example. Since we reduced the problem of checking $\mathcal{D} \models \varphi$ to checking the feasibility of $\mathcal{D} \| \mid T_{\neg \varphi}$, such a witness can be provided by a computation of the combined FDS $\mathcal{D} \| T_{\neg \varphi}$.

In the following we present an algorithm which produces a computation of an FDS that has been declared feasible. We introduce the list data structure to represent a linear list of states. We use $\Lambda$ to denote the empty list. For two lists $L_{1}=\left(s_{1}, \ldots, s_{a}\right)$ and $L_{2}=\left(s_{b}, \ldots, s_{c}\right)$, we denote by $L_{1} * L_{2}$ their concatenation, defined by $L_{1} * L_{2}=\left(s_{1}, \ldots, s_{a}, s_{b} \ldots, s_{c}\right)$. For a non-empty predicate $U \subseteq \Sigma$, we denote by $\operatorname{choose}(U)$ a consistent choice of one of the members of $U$. 
The function path(source, destination, $R$ ), presented in Fig. 2, returns a list which contains the shortest $R$-path from a state in source to a state in destination. In the case that source and destination have a non-empty intersection, path will return a state belonging to this intersection which can be viewed as a path of length zero.

Function path(source, destination : predicate; $R$ : relation) : list -

- Compute shortest path from source to destination

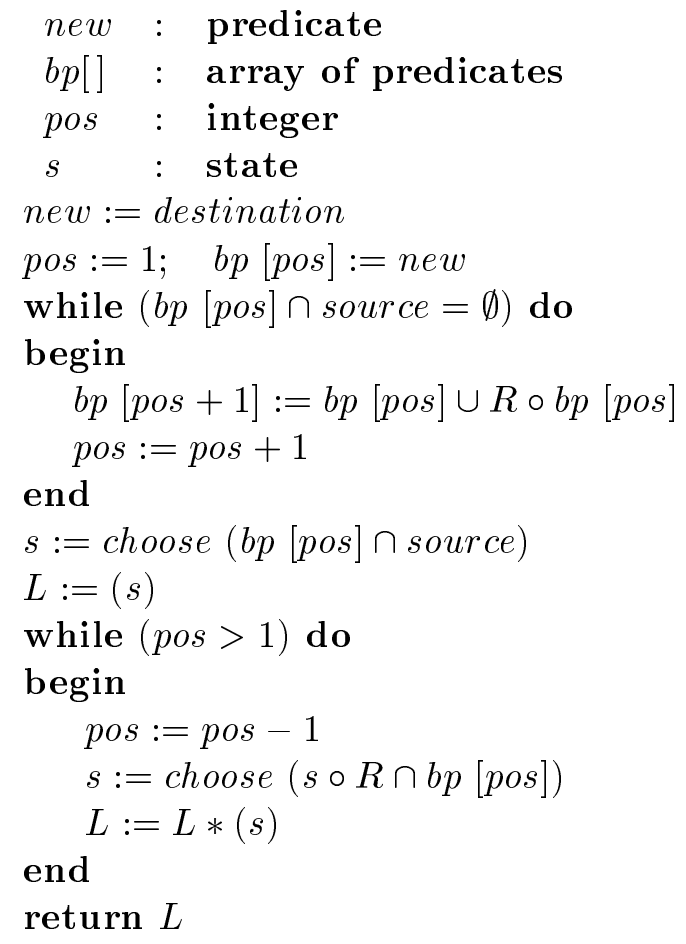

Figure 2: Function path.

Finally, in Fig. 3 we present an algorithm which produces a computation of a given FDS. Although a computation is an infinite sequence of states, if $\mathcal{D}$ is feasible, it always has an ultimately periodic computation of the following form:

$$
\sigma: \underbrace{s_{0}, s_{1}, \ldots, s_{k}}_{\text {prefix }}, \underbrace{s_{k+1}, \ldots, s_{k}}_{\text {period }}, \underbrace{s_{k+1}, \ldots, s_{k}}_{\text {period }}, \cdots, \underbrace{s_{k+1}, \ldots, s_{k}}_{\text {period }}, \cdots
$$

Based on this observation, our witness extracting algorithm will return as result the two finite sequences prefix and period.

The algorithm starts by checking whether FDS $\mathcal{D}$ is feasible. It uses Algorithm fEAsiBle to perform this check. If $\mathcal{D}$ is found to be infeasible, the algorithm exits while providing a pair of empty lists as a result.

If $\mathcal{D}$ is found to be feasible, we store in final the set of states returned by FEASIBLE. This set contains the set of all states participating in a computation of $\mathcal{D}$. We restrict the transition relation $R$ to pairs $\left(s_{1}, s_{2}\right)$ where both $s_{1}$ and $s_{2}$ are states within final. Next, we perform a search for a terminal maximal strongly connected subgraph (terminal MSCS) within final. The search starts at $s \in$ final, an arbitrarily chosen state within final. In the loop at lines 5 and 6 we search for a state $s$ 
Algorithm witness $(\mathcal{D}):[$ list, list $]$ — Extract a witness for a feasible FDS.

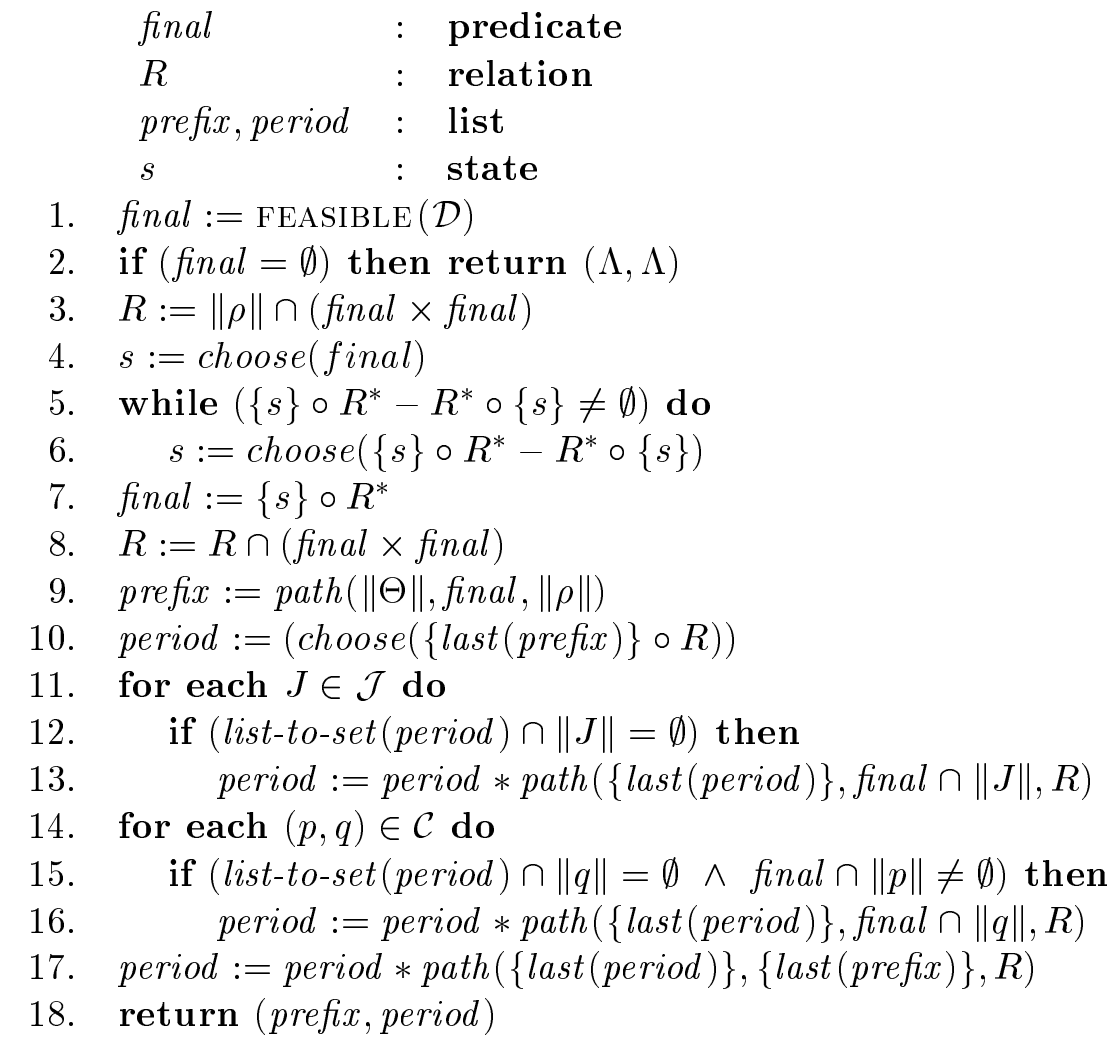

Figure 3: Algorithm WITNESS.

satisfying $\{s\} \circ R^{*} \subseteq R^{*} \circ\{s\}$. i.e. a state all of whose $R^{*}$-successors are also $R^{*}$-predecessors. This is done by successively replacing $s$ by a state $s \in\{s\} \circ R^{*}-R^{*} \circ\{s\}$, as long as the set of $s$-successors is not contained in the set of $s$-predecessors. Eventually, execution of the loop must terminate when $s$ reaches a terminal MSCS within final. Termination is guaranteed because each such replacement moves the state from one MSCS to a proceeding MSCS in the canonical decomposition of final into MSCS's.

A central point in the proof of correctness of Algorithm FEASIBLE established that any terminal MSCS within final is a fair subgraph. Line 7 computes the MSCS containing $s$ and assigns it to the variable final, while line 8 restricts the transition relation $R$ to edges connecting states within final. Line 9 draws a (shortest) path from an initial state to the subgraph final.

Lines 10 - 17 construct in period a traversing path, starting at the last state of the prefix, last(prefix), and returning to the same state, while visiting on the way states that ensure that an infinite repetition of the period will fulfill all the fairness requirements.

Lines 11-13 ensure that period contains a $J$-state, for each $J \in \mathcal{J}$. To prevent unnecessary visits to states, we extend the path to visit the next $J$-state only if the part of period that has already been constructed did not visit any $J$-state. Lines 14-16 similarly take care of compassion. Here we extend the path to visit a $q$-state only if the constructed path did not already do so and the MSCS final contains some $p$-state. Finally, in line 17, we complete the path to form a closed cycle by looping back to last(prefix). 


\section{Symbolic Model Checking CTL* Properties}

In the following, we show that algorithm FEASIBLE can be used to model check an arbitrary CTL* formula over a finite state FDS, taking weak and strong fairness constraints into consideration. We define $\mathrm{CTL}^{*}$ with both future and past temporal operators. We denote the fragment of $\mathrm{CTL}^{*}$ without the past operators as the future fragment of $\mathrm{CTL}^{*}$.

An enumerative algorithm for model checking the future fragment of $\mathrm{CTL}^{*}$ is presented in [EL87]. In this work, Emerson and Lei show that model checking a CTL* formula over a finite state system, can be performed by recursive calls to an LTL model checker. We take a similar approach, using algorithm FEASIBLE to verify an arbitrary $\mathrm{CTL}^{*}$ formula over a finite state FDS $\mathcal{D}$.

In the following, we first present the syntax and semantics of the logic, then discuss the use of algorithm FEASIBLE for verifying CTL* properties.

\subsection{The Logic CTL*}

A propositional $\mathrm{CTL}^{*}$ formula is constructed out of propositions to which we apply the boolean operators, temporal operators and path quantifiers. The temporal operators are the same operators presented in section 3 for LTL. The path quantifiers are $E_{f}$ and $A_{f}$, as defined below.

A CTL* formula $p$ is interpreted over the state graph (Kripke structure) generated by an FDS $\mathcal{D}$. In the following, we use the term path in $\mathcal{D}$ as synonymous to a computation of $\mathcal{D}$.

There are two types of formulas in $\mathrm{CTL}^{*}$ : State formulas which are interpreted over states and path formulas which are interpreted over paths. Let $\mathcal{P}$ be a finite set of propositions. The syntax of a $\mathrm{CTL}^{*}$ formula is defined inductively as follows.

State formulas:

- Every proposition $p \in \mathcal{P}$ is a state formula.

- If $p$ is a path formula, then $E_{f} p$ and $A_{f} p$ are state formulas.

- If $p$ and $q$ are state formulas then so are $\neg p$ and $p \vee q$.

Path formulas:

- Every state formula is a path formula.

- If $p$ and $q$ are path formulas then so are $\neg p, p \vee q, \bigcirc p, p \mathcal{U} q, \ominus p$ and $p \mathcal{S} q$.

The formulas of $\mathrm{CTL}^{*}$ are all the state formulas generated by the above rules.

A state formula of the form $Q p$, where $Q$ is a path quantifier and $p$ is a path formula containing no path quantifiers is called a basic state formula. A basic state formula of the form $A_{f} \psi\left(E_{f} \psi\right)$ is called a basic universal (existential) formula. Note that the set of basic universal formulas corresponds to the set of linear temporal logic formulas (LTL).

The semantics of a $\mathrm{CTL}^{*}$ formula is defined inductively as follows. State formulas are interpreted over states in $\mathcal{D}$. We define the notion of a path formula $p$ holding at a state $s$ in $\mathcal{D}$, denoted $(\mathcal{D}, s) \models p$, as follows:

- For an assertion $p$,

$$
(\mathcal{D}, s) \models p \quad \Leftrightarrow \quad s \models p
$$

- $(\mathcal{D}, s) \models \neg p \quad \Leftrightarrow \quad(\mathcal{D}, s) \not \models p$

- $(\mathcal{D}, s) \models p \vee q \quad \Leftrightarrow \quad(\mathcal{D}, s) \models p$ or $(\mathcal{D}, s) \models q$

- $(\mathcal{D}, s) \models E_{f} p \quad \Leftrightarrow \quad(\mathcal{D}, \pi, j) \models p$ for some path $\pi=\pi_{0}, \pi_{1}, \ldots \in \mathcal{C} \operatorname{omp}(\mathcal{D})$, and position $j \geq 0$ satisfying $\pi_{j}=s$.

The universal path quantifier is defined by $A_{f} p=\neg E_{f} \neg p$. Note that we have only defined the fair versions $A_{f}$ and $E_{f}$ of the path quantifiers. If one wants to verify a formula $A \varphi$ over an FDS $\mathcal{D}$, 
it can be done by verifying $A_{f} \varphi$ over $\mathcal{D}_{\text {unfair }}$, which is obtained from $\mathcal{D}$ by removing all fairness requirements.

Path formulas are interpreted over a path (computation) in $\mathcal{D}$. We define the notion of a path formula $p$ holding at position $j \geq 0$ of a path $\pi$ in $\mathcal{C} o m p(\mathcal{D})$, denoted $(\mathcal{D}, \pi, j) \models p$, as follows:

- For a state formula $p$,

$$
\begin{aligned}
& (\mathcal{D}, \pi, j) \models p \quad \Leftrightarrow \quad(\mathcal{D}, s) \models p \text {, for } s=\pi_{j} \\
& \text { - }(\mathcal{D}, \pi, j) \models \neg p \quad \Leftrightarrow \quad(\mathcal{D}, \pi, j) \not \models p \\
& \text { - }(\mathcal{D}, \pi, j) \models p \vee q \quad \Leftrightarrow \quad(\mathcal{D}, \pi, j) \models p \text { or }(\mathcal{D}, \pi, j) \models q \\
& \text { - }(\mathcal{D}, \pi, j) \models \bigcirc p \quad \Leftrightarrow \quad(\mathcal{D}, \pi, j+1) \mid p \\
& \text { - } \quad(\mathcal{D}, \pi, j) \models p \mathcal{U} q \quad \Leftrightarrow \quad(\mathcal{D}, \pi, k) \models q \text { for some } k \geq j \\
& \text { and }(\mathcal{D}, \pi, i) \models p \text { for every } i, j \leq i<k \\
& \text { - }(\mathcal{D}, \pi, j) \models \ominus p \quad \Leftrightarrow \quad j>0 \text { and }(\mathcal{D}, \pi, j-1) \models p \\
& \text { - }(\mathcal{D}, \pi, j) \models p \mathcal{S} q \quad \Leftrightarrow \quad(\mathcal{D}, \pi, k) \models q \text { for some } k, 0 \leq k \leq j \\
& \text { and }(\mathcal{D}, \pi, i) \models p \text { for every } i, k<i \leq j
\end{aligned}
$$

Let $p$ be a CTL $^{*}$ formula. We say that $p$ holds on $\mathcal{D}(p$ is $\mathcal{D}$-valid $)$, denoted $\mathcal{D} \models p$, if $(\mathcal{D}, s) \models p$, for every initial state $s$ in $\mathcal{D}$. A CTL* formula $p$ is called satisfiable if it holds on some model $\mathcal{D}$. A $\mathrm{CTL}^{*}$ formula is called valid if it holds on all models. Let $p$ and $q$ be $\mathrm{CTL}^{*}$ formulas. We use the notations $p \Longrightarrow q$ and $p \Longleftrightarrow q$ as a shorthand for $A_{f} \square(p \rightarrow q)$ and $A_{f} \square(p \leftrightarrow q)$ respectively.

\subsection{Model Checking Basic State Formulas}

In the following we show how to model check basic state formulas over an FDS $\mathcal{D}$.

Algorithm FEASIBLE is formulated in set-theoretic terms. However, its implementation obviously represents any set of states by a BDD which also represents an assertion. In the following we use notations such as $\|\alpha\|=\operatorname{FEASIBLE}(\mathcal{D})$ in order to refer to the assertion $\alpha$ characterizing the set of states returned by the algorithm.

In Fig. 4 we present algorithm SAT-E $\mathrm{E}_{f}$ which evaluates the set of $\mathcal{D}$-reachable states satisfying a basic existential formula $E_{f} \varphi$. Note that, since $\varphi$ is a path formula with no embedded path quantifiers, it is also an LTL formula, for which a tester can be constructed.

\section{Algorithm $\operatorname{SAT}_{-}(\mathcal{D}, \varphi)$ : predicate -}

- Compute the set of $\mathcal{D}$-reachable states satisfying $E_{f} \varphi$

1. Define the temporal tester $T_{\varphi}$ for $\varphi$.

2. Define the synchronous parallel composition $\mathcal{D} \| T_{\varphi}$.

3. Evaluate $\|\psi\|=\|\chi(\varphi)\| \cap \operatorname{FEASIBLE}\left(\mathcal{D} \| T_{\varphi}\right)$

4. Project away the auxiliary variables $X_{\varphi}$ of $T_{\varphi}$, returning $\left\|\alpha_{E \varphi}\right\|=\left\|\exists X_{\varphi}: \psi\right\|$.

Figure 4: Algorithm SAT-E $_{f}$

The following claim states that the set $\left\|\alpha_{E_{\varphi}}\right\|$ evaluated by algorithm SAT-E ${ }_{f}$ is exactly the set of $\mathcal{D}$-reachable states satisfying the CTL ${ }^{*}$ formula $E_{f} \varphi$.

Claim $9 \quad(\mathcal{D}, s) \models E_{f} \varphi \quad$ iff $\quad s \in\left\|\alpha_{E_{\varphi}}\right\|$

Corollary $10 \mathcal{D}=\alpha_{E_{\varphi}} \Longleftrightarrow E_{f} \varphi$ 
Let $\mathcal{D}$ be an FDS and $A_{f} \varphi$ be a basic universal formula. To evaluate the set of $\mathcal{D}$-reachable states satisfying the formula, we use the $\mathrm{CTL}^{*}$ congruence $A_{f} \varphi \Longleftrightarrow \neg E_{f} \neg \varphi$.

In Fig. 5 we present algorithm SAT-A $f$ which evaluates the set of $\mathcal{D}$-reachable states satisfying a basic universal formula $A_{f} \varphi$.

Algorithm SAT-A $f(\mathcal{D}, \varphi)$ : predicate -

- C Compute the set of $\mathcal{D}$-reachable states, satisfying $A_{f} \varphi$

1. Define the temporal tester $T_{\neg \varphi}$.

2. Define the synchronous parallel composition $\mathcal{D} \| T_{\neg \varphi}$.

3. Evaluate $\|\psi\|=\|\chi(\neg \varphi)\| \cap \operatorname{FEASIBLE}\left(\mathcal{D} \| T_{\neg \varphi}\right)$

4. Project away the auxiliary variables of $T_{\neg \varphi}$ and complement the result, returning $\left\|\alpha_{A \varphi}\right\|=\left\|\neg \exists X_{\neg \varphi}: \psi\right\|$.

Figure 5: Algorithm SAT-A $f$

The following claim states that the set $\left\|\alpha_{A_{\varphi}}\right\|$ evaluated by algorithm SAT-A $f$ is exactly the set of $\mathcal{D}$-reachable states satisfying the $\mathrm{CTL}^{*}$ formula $A_{f} \varphi$.

Claim $11(\mathcal{D}, s) \models A_{f} \varphi \quad$ iff $\quad s \in\left\|\alpha_{A_{\varphi}}\right\|$

Corollary $12 \mathcal{D}=\alpha_{A_{\varphi}} \Longleftrightarrow A_{f} \varphi$

The two algorithms $\operatorname{SAT}^{-E_{f}}$ and $\operatorname{SAT}_{-} \mathrm{A}_{f}$ can be combined into a single algorithm $\operatorname{SAT}-\operatorname{BASIC}(\mathcal{D}, \varphi)$, defined by

$$
\begin{aligned}
& \operatorname{SAT-BASIC}\left(\mathcal{D}, E_{f} \varphi\right)=\operatorname{SAT}_{f}(\mathcal{D}, \varphi) \\
& \operatorname{SAT-BASIC}\left(\mathcal{D}, A_{f} \varphi\right)=\operatorname{SAT}_{f}(\mathcal{D}, \varphi)
\end{aligned}
$$

\subsection{Decomposing an Arbitrary CTL* Formula into Basic Formulas}

Consider an arbitrary (non basic) $\mathrm{CTL}^{*}$ formula $p$ which we wish to verify over an FDS $\mathcal{D}$. Following [EL87], we reduce the task of verifying formula $p$ into simpler subtasks, each required to verify a basic state formula over $\mathcal{D}$.

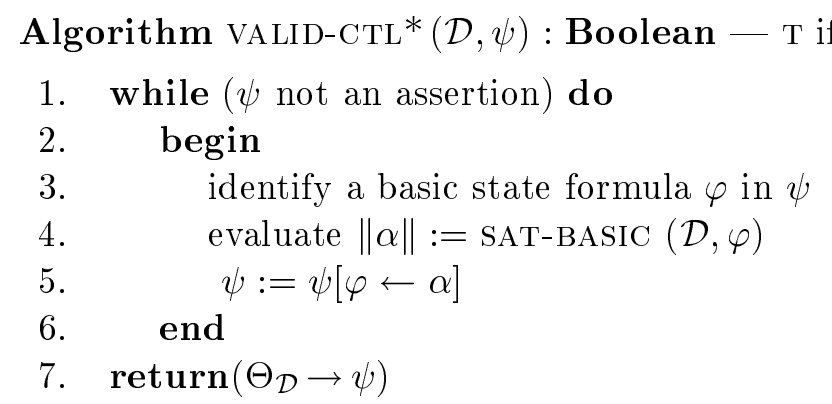

Figure 6: Algorithm VALID-CTL* 
In Fig. 6 we present algorithm VALID-CTL* for the verification of an arbitrary CTL* formula $\psi$ over an FDS $\mathcal{D}$. The algorithm consists of a while loop, where at each iteration of the loop formula $\psi$ is reduced to a new formula in which all occurrences of some basic state formula $\varphi$ within $\psi$, are replaced by an assertion congruent to $\varphi$. The loop terminates when $\psi$ is reduced to an assertion $\widetilde{\psi}$ that characterizes the set of states in $\mathcal{D}$ satisfying $\psi$. Formula $\psi$ is $\mathcal{D}$-valid if $\left\|\Theta_{\mathcal{D}}\right\| \subseteq\|\widetilde{\psi}\|$, or equivalently $\Theta_{\mathcal{D}} \rightarrow \widetilde{\psi}$. The following claim states the soundness of algorithm VALID-CTL*.

Let $\mathcal{D}$ be an FDS and $\psi$ be an arbitrary $\mathrm{CTL}^{*}$ formula, then

Claim $13 \mathcal{D}=\psi \quad$ iff $\quad$ VALID-CTL $*(\mathcal{D}, \psi)$ returns $\mathrm{T}$.

\section{Example}

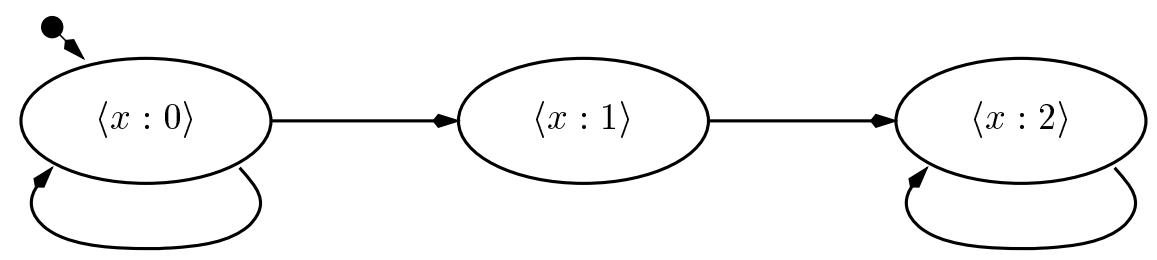

Figure 7: An example system $\mathcal{D}$

Consider the system $\mathcal{D}$ presented in Fig. 7. This system has a single state variable $x$ and no fairness requirements. For this system we wish to prove the property $f: E_{f} \square E_{f} \diamond(x=1)$, claiming the existence of a computation from each of whose states it is possible to reach a state at which $x=1$.

Using algorithm VALID-CTL*, the task of verifying the non-basic formula

$$
E_{f} \square E_{f} \diamond(x=1)
$$

is reduced into the following tasks:

R1. Evaluate $\left\|\alpha_{1}\right\|=\operatorname{SAT-BASIC}\left(\mathcal{D}, E_{f} \diamond(x=1)\right)$. This yields $\alpha_{1}:(x=0)$.

R2. Evaluate $\left\|\alpha_{2}\right\|=\operatorname{SAT-BASIC}\left(\mathcal{D}, E_{f} \square \alpha_{1}\right)$. This yields $\alpha_{2}:(x=0)$.

R3. Evaluate $\Theta_{\mathcal{D}} \rightarrow \alpha_{2}$. This yields T.

\section{Experimental Results}

The algorithms described in this paper were implemented within the TLV system [PS96]. In the following section, we summarize our experimental results for algorithm FEASIBLE. The experiments were carried on a Sun Ultra with 1 Gigabyte of memory. We limit our attention to LTL properties since our intention is to test the performance of algorithm FEASIBLE, which is the same for LTL and CTL*.

\subsection{Compassion at the Algorithmic Level}

In order to test whether compassion at the algorithmic level yields better performance, we verify several examples which require compassion, using three different verification methods: 
1. Verifying compassion at the algorithmic level. We denote this methods by NT (no transformation).

2. Transforming compassion to justice. From an automata theoretic perspective this transforms a Streett automata into a Büchi automata. We denote this method by CJ (compassion transformed to justice).

3. Replacing compassion requirements in the system by adding an antecedent to the verified property. We denote this method by CA (compassion as antecedent).

In method CJ, every compassion requirement is transformed into a justice requirement, at the price of introducing an extra boolean variable. Let $\mathcal{D}:\langle V, \Theta, \rho, \mathcal{J}, \mathcal{C}\rangle$ be an FDS. For every $\left\langle p_{i}, q_{i}\right\rangle \in \mathcal{C}$ we introduce a new variable $r_{i}$ and modify $\mathcal{D}$ as follows:

$$
\begin{aligned}
\widetilde{V} & : V \cup\left\{r_{i}\right\} \\
\widetilde{\Theta} & : \Theta \wedge\left(r_{i}=\mathrm{F}\right) \\
\widetilde{\rho} & : \rho \wedge\left(r_{i} \rightarrow\left(\neg p_{i} \wedge r_{i}^{\prime}\right)\right) \\
\widetilde{\mathcal{J}} & : \mathcal{J} \cup\left\{\left(r_{i} \vee q_{i}\right)\right\} \\
\widetilde{\mathcal{C}} & : \mathcal{C}-\left\{\left(p_{i}, q_{i}\right)\right\}
\end{aligned}
$$

Method CA replaces a set of compassion requirements by a conjunction of LTL formulas, added as an antecedent to the verified property. Let $\mathcal{D}$ be an FDS, $\varphi$ be a formula we wish to verify over $\mathcal{D}$ and $\mathcal{C}$ be the set of compassion requirements in $\mathcal{D}$. We replace the verification of $\mathcal{D}=\varphi$ by the following verification task:

$$
\mathcal{D}_{(-\mathcal{C})} \models\left(\bigwedge_{\langle p, q\rangle \in \mathcal{C}}(\square \diamond p \rightarrow \square \diamond q)\right) \rightarrow \varphi
$$

where $\mathcal{D}_{(-\mathcal{C})}$ is the FDS $\mathcal{D}$ from which all the compassion requirements have been removed.

Note that a similar method can be applied to $\mathrm{CTL}^{*}$ formulas. Let $\psi$ be a CTL* property, and $c$ be defined as follows:

$$
c=\bigwedge_{\langle p, q\rangle \in \mathcal{C}}(\square \diamond p \rightarrow \square \diamond q)
$$

We modify the calls to SAT- $\mathrm{E}_{f}$ and $\mathrm{SAT}^{-\mathrm{A}_{f}}$ in algorithm VALID-CTL* as follows:

- For a basic existential formula, call $\mathrm{SAT}_{-} \mathrm{E}_{f}\left(\mathcal{D}_{(-\mathcal{C})}, c \wedge \varphi\right)$.

- For a basic universal formula, call $\mathrm{SAT}_{-\mathrm{A}_{f}}\left(\mathcal{D}_{(-\mathcal{C})}, c \rightarrow \varphi\right)$.

\subsubsection{Feasibility of Parameterized Programs}

The programs we use for experimentation are parameterized programs, of the form

$$
S(n): P[1]\|P[2]\| \ldots \| P[n]
$$

which are verified for different values of $n$.

Consider program DINE presented in Fig. 8. This program is a symmetric solution to the dining philosophers problem, using semaphores for coordination between processes. Program DINE satisfies 
the safety requirement of mutual exclusion, stating that no neighboring philosophers can dine at the same time. However, this program fails to satisfy the liveness requirement of accessibility for the first process, stating that if the philosopher wishes to dine, it will eventually do so, as specified by:

$$
\psi_{1}: \square\left(a t_{-} \ell_{2}[1] \rightarrow \diamond a t_{-} \ell_{4}[1]\right)
$$

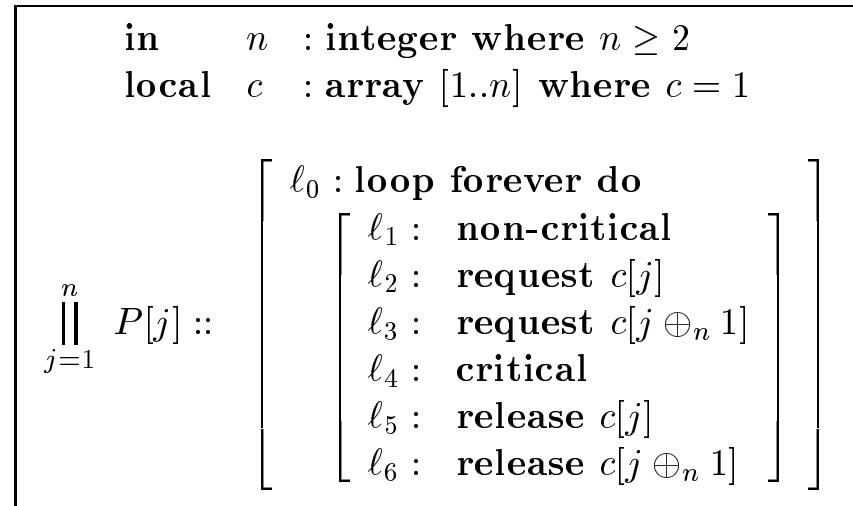

Figure 8: Program DINE: The Dining Philosophers.

Program DINE is written in SPL (Simple Programming Language). The process of translating an SPL program to an FDS can be done automatically [MP95]. The translation associates a compassion requirement with every request statement. The execution of the statement request $s$ reduces the value of the semaphore $s$ by 1 . The statement can be executed only if $s>1$. For example, the compassion requirement associated with $\ell_{2}$ of process 1 is $\left\langle a t_{-} \ell_{2}[1] \wedge c[1]=1, a t_{-} \ell_{3}[1]\right\rangle$. This requirement ensures that if statement $\ell_{2}$ is infinitely often enabled, it is infinitely often taken.

The non-critical statement has no corresponding justice or compassion requirement. A process may remain indefinitely in such a statement. For all other statements, the translation associates a justice requirement of the form $\neg a t_{-} \ell_{i}$. In program DINE, statements $\ell_{0}, \ell_{4}, \ell_{5}$ and $\ell_{6}$ each have corresponding justice requirements.

Note that it may be possible to translate an SPL program to an FDS using fewer justice requirements, however, this typically requires a deeper understanding of the program, and thus manual translation.

As a second example we consider the asymmetric version of the dining philosophers, program DINE-CONTR (dining philosophers with one contrary process), where the behavior of one of the philosophers is reversed, i.e. it first lifts the right fork and then the left one. The accessibility property $\psi$ is valid for program DINE-CONTR.

The third example is program MUX-SEM, presented in Fig. 9, implements mutual exclusion by semaphores. The following accessibility property is valid for program MUX-SEM:

$$
\psi_{2}: \square\left(a t_{-} \ell_{2}[1] \rightarrow \diamond a t_{-} \ell_{3}[1]\right)
$$

In the following experiments we verify the accessibility property $\psi_{1}$ for program DINE and DINE-CONTR, and the accessibility property $\psi_{2}$ for program MUX-SEM.

In the tables summarizing our results, we use the following notations: 


\begin{tabular}{|l}
$\begin{array}{lll}\text { in } & n & : \text { integer where } n>0 \\
\text { local } & y & : \text { integer where } y=1\end{array}$ \\
$\prod_{i=1}^{n} P[i]::$ \\
{$\left[\begin{array}{ll}\ell_{0}: \text { loop forever do } \\
{\left[\begin{array}{ll}\ell_{1}: & \text { non-critical } \\
\ell_{2}: & \text { request } y \\
\ell_{3}: & \text { critical } \\
\ell_{4}: & \text { release } y\end{array}\right]}\end{array}\right]$}
\end{tabular}

Figure 9: Program MUX-SEM: Mutual Exclusion with Semaphores.

- $\mathbf{n}$ - The number of processes for which the parameterized program has been tested.

- Proc. - The verification method used for the compassion requirements.

- $|\mathcal{J}|,|\mathcal{C}|$ - The number of justice and compassion requirements in the FDS $\mathcal{D} \| \mid T_{\neg \varphi}$.

- Time - Timing results (in seconds).

- BDD Peak - the maximum number of allocated BDD nodes.

- Op. - The number of pre-composition (pre-image) operations invoked by algorithm FEASIBLE.

- Ext. Iter. - The number of external while-loop iterations performed ( lines 4-11 of algorithm FEASIBLE ).

\begin{tabular}{|r|r|r|r|r|r|r|r|}
\hline $\mathrm{n}$ & Proc. & $|\mathcal{J}|$ & $|\mathcal{C}|$ & Time & BDD Peak & Op. & Ext. Iter. \\
\hline \hline \multirow{3}{*}{3} & NT & 14 & 6 & 0.49 & 10016 & 474 & 4 \\
\cline { 2 - 8 } & CJ & 20 & 0 & 5.34 & 42674 & 711 & 5 \\
\cline { 2 - 8 } & CA & 37 & 0 & 36.79 & 414467 & 1793 & 4 \\
\hline \hline \multirow{3}{*}{4} & NT & 18 & 8 & 3.54 & 17146 & 1007 & 6 \\
\cline { 2 - 8 } & CJ & 26 & 0 & 148.02 & 223395 & 1079 & 5 \\
\cline { 2 - 8 } & CA & 49 & 0 & 3829.80 & 2769339 & 2849 & 4 \\
\hline
\end{tabular}

Table 1: Program DINE

The overall numbers of fairness requirements $|\mathcal{J}|+|\mathcal{C}|$ of the NT and CJ methods are equal, but the compassion requirements of method NT have been transformed to justice requirements in method CJ. The additional justice requirements in method CA are due to the tester, which is generated from a more complex property.

In all our experiments on real systems, method NT has the best results and method CA has (by far) the worst results, both in execution time, and memory consumption. However, in some cases the number of pre-composition operations for method CJ was less than that of method NT. 


\begin{tabular}{|r|r|r|r|r|r|r|r|}
\hline $\mathrm{n}$ & Proc. & $|\mathcal{J}|$ & $|\mathcal{C}|$ & Time & BDD Peak & Op. & Ext. Iter. \\
\hline \hline \multirow{3}{*}{3} & NT & 14 & 6 & 0.98 & 10016 & 991 & 10 \\
\cline { 2 - 8 } & CJ & 20 & 0 & 2.74 & 36687 & 382 & 5 \\
\cline { 2 - 8 } & CA & 37 & 0 & 19.40 & 334345 & 985 & 4 \\
\hline \hline \multirow{3}{*}{4} & NT & 18 & 8 & 3.63 & 21112 & 1119 & 9 \\
\cline { 2 - 8 } & CJ & 26 & 0 & 29.11 & 135478 & 693 & 6 \\
\cline { 2 - 8 } & CA & 49 & 0 & 1670.29 & 2066671 & 1567 & 4 \\
\hline
\end{tabular}

Table 2: Program DINE-CONTR

\begin{tabular}{|r|r|r|r|r|r|r|r|}
\hline $\mathrm{n}$ & Proc. & $|\mathcal{J}|$ & $|\mathcal{C}|$ & Time & BDD Peak & Op. & Ext. Iter. \\
\hline \hline 3 & NT & 11 & 3 & 0.09 & 5007 & 168 & 5 \\
\cline { 2 - 8 } & CJ & 14 & 0 & 0.23 & 10002 & 162 & 5 \\
\cline { 2 - 8 } & CA & 22 & 0 & 0.67 & 22805 & 184 & 3 \\
\hline \hline \multirow{4}{*}{4} & NT & 14 & 4 & 0.16 & 8726 & 204 & 5 \\
\cline { 2 - 8 } & CJ & 18 & 0 & 0.59 & 14574 & 198 & 5 \\
\cline { 2 - 8 } & CA & 29 & 0 & 4.40 & 98191 & 287 & 3 \\
\hline \hline 5 & NT & 17 & 5 & 0.26 & 10000 & 240 & 5 \\
\cline { 2 - 8 } & CJ & 22 & 0 & 1.08 & 21202 & 234 & 5 \\
\cline { 2 - 8 } & CA & 36 & 0 & 61.11 & 336517 & 405 & 3 \\
\hline \hline 6 & NT & 20 & 6 & 0.41 & 10051 & 276 & 5 \\
\cline { 2 - 7 } & CJ & 26 & 0 & 1.80 & 30369 & 270 & 5 \\
\cline { 2 - 7 } & CA & 43 & 0 & 1296.68 & 1070510 & 540 & 3 \\
\hline
\end{tabular}

Table 3: Program MUX-SEM

\subsubsection{Feasibility of Randomly Generated Graphs}

The experiments for real systems were not conclusive with regard to the comparison between methods NT and CJ. Although method NT has better performance than CJ, the latter sometimes requires significantly fewer pre-composition operations. In this section we present additional experiments for comparing between the two methods.

We can view a system as a digraph where the state space of the system corresponds to the vertex space, and there is a directed edge from $u$ to $v$ in the digraph iff there is a transition from $u$ to $v$ in the system.

The problem in performing experiments over any set of real examples is that they provide a limited range of digraph patterns. Our experiments should not be biased towards any specific design. Therefore, we performed numerous experiments on random digraphs. We generate random digraphs as suggested in [Yan99].

Given a graph $G$ with vertices $V$ and edges $E$, the order of $G$ is $n=|V|$ and the density of $G$ is $d=|E| /|V|$.

In the following tables, $n=1000$. Each table only changes one parameter, either the size of the set of compassion requirements, or the density of the random graph. Each table entry is the 
average of 100 experiments.

\begin{tabular}{|c|rrr|rrr|}
\hline$|\mathcal{C}|$ & \multicolumn{3}{|c|}{ NT } & \multicolumn{3}{c|}{ CJ } \\
\cline { 2 - 7 } & Time & Op. & Ext. Iter. & Time & Op. & Ext. Iter. \\
\hline 40 & 1.33 & 285.1 & 2.3 & 1.14 & 83.1 & 2.0 \\
\hline 60 & 1.61 & 382.7 & 2.3 & 1.67 & 92.6 & 2.0 \\
\hline 80 & 1.29 & 301.7 & 2.2 & 1.97 & 79.0 & 2.0 \\
\hline 100 & 1.89 & 512.7 & 2.4 & 19.46 & 148.5 & 2.0 \\
\hline 120 & 2.90 & 813.1 & 2.5 & 105.48 & 216.9 & 2.0 \\
\hline
\end{tabular}

Table 4: Varying compassion set size. $\mathrm{d}=1.5$.

\begin{tabular}{|c|rrr|rrr|}
\hline \multirow{2}{*}{ Density } & \multicolumn{3}{|c|}{ NT } & \multicolumn{3}{c|}{ CJ } \\
\cline { 2 - 7 } & Time & Op. & Ext. Iter. & Time & Op. & Ext. Iter. \\
\hline 1.2 & 0.16 & 52.9 & 2.1 & 0.19 & 20.1 & 2.0 \\
\hline 1.6 & 1.92 & 312.9 & 2.3 & 1.32 & 78.2 & 2.0 \\
\hline 2 & 8.49 & 780.3 & 2.5 & 4.03 & 159.0 & 2.0 \\
\hline 2.4 & 13.25 & 882.6 & 2.5 & 5.90 & 171.4 & 2.0 \\
\hline 2.8 & 18.54 & 1026.9 & 2.6 & 7.89 & 200.5 & 2.0 \\
\hline
\end{tabular}

Table 5: Varying density. $|\mathcal{C}|=40$.

Table 4 produces results similar to those obtained for real systems. The real systems we checked were parameterized, with compassion requirements associated with each process. Therefore, the number of compassion requirements increases together with the number of processes. In method CJ, each requirement introduces an additional variable, which affects performance of pre-composition operations. Therefore, when the number of pre-composition operations is roughly the same, using method NT is preferable.

In Table 5 method CJ requires less time and less pre-composition operations as density increases. The reduced number of pre-composition operations compensates for the performance penalty of each pre-composition operation.

\subsubsection{Conclusions}

In many cases, the best results for time and memory are obtained in method NT (Compassion at the algorithmic level). Method NT is likely to be better for systems with big compassion sets. Method CJ requires an addition of a program variable for each compassion requirement. This decreases the performance of pre-composition operations, and is the reason that even though in some cases where the number of pre-composition operations is lower in method CJ, the performance is still worse since each pre-composition operations cost more.

By far, the worst results occured using method CA, where compassion is added as an antecedent of the property. The tester generated for such modified specifications has four additional variables, one for each principle temporal operator in the formula $(\square \diamond p \rightarrow \square \diamond q)$, and also has additional justice requirements. These account for the decreased performance. 


\subsection{Reducing the Number of Justice Conditions}

When we define the FDS associated with a given program, we introduce a justice requirement for each of the program locations, to ensure that the process does not remain in that location indefinitely. However, this creates many justice conditions which may slow the algorithm down. In the following, we verify the effect of reducing the number of justice requirements, on the performance of algorithm FEASIBLE.

Fig. 10 presents a toy example designed to allow us to create an FDS which corresponds to the program, such that the number of justice requirements is greatly reduced when compared to the FDS which would normally be generated. Each process of program CYCLE may remain at location $\ell_{1}$ indefinitely. However, once the process executes $\ell_{1}$ it must not remain stuck in one of the other program locations, rather, it should cycle through the rest of the program locations until it returns to $\ell_{1}$.

The idle statement makes a nondeterministic choice between either remaining forever in the current program location, or advancing to the next statement. The skip statement does nothing except to advance to the next statement.

$$
\|_{j=1}^{n} P[j]::\left[\begin{array}{c}
\ell_{0}: \text { loop forever do } \\
{\left[\begin{array}{ll}
\ell_{1}: & \text { idle } \\
\ell_{2}: & \text { skip } \\
\vdots & \\
\ell_{m}: & \text { skip }
\end{array}\right]}
\end{array}\right]
$$

Figure 10: Program CYCLE.

For each process $i$, program CYCLE has program locations $\ell_{0}, \ldots, \ell_{m}$. The standard FDS corresponding to program CYCLE contains, for each process $i$ and for each $0 \leq j \leq m, j \neq 1$, a justice condition of the form $\neg a t \ell_{j}$. Therefore, a program of $p$ processes and $m$ locations in each process has $p *(m-1)$ justice conditions. In the modified FDS each process $i$ has only a single justice condition: $a t_{-} \ell_{1}$.

We verify the following accessibility property, for the first process:

$$
\psi_{3}: \square\left(a t_{-} \ell_{2} \rightarrow \diamond a t_{-} \ell_{m}\right)
$$

Tables 6 and 7 compare executions of programs with 6 and 10 program locations. These tables show a slight improvement when less justice conditions are generated. However, program CYCLE was tailored to simplify the reduction of justice conditions. In other programs we have checked, such as DINE, the price of reducing the number of justice conditions was either adding a single, complex justice requirement, or adding variables to the verified system. In these cases, reducing the number of justice requirements increased execution time of the feasibility algorithm.

Note that for justice requirements of the form $\neg a t_{-} \ell_{i}$, line 9 of algorithm FEASIBLE converges in only two steps. Although the standard, automatic way for generating an FDS from a program produces more justice requirements than what could optimally be produced by hand, the price for processing each of these justice requirement is small. This can explain our experimental results.

We thus conclude that reducing the number of justice conditions is usually not recommended. 
Table 6: Six Program Locations

\begin{tabular}{|c|c|c|}
\hline $\mathrm{N}$ & 5 Justice Cond. & 1 Justice Cond. \\
\hline 10 & 0.57 & 0.26 \\
\hline 14 & 4.74 & 4.44 \\
\hline 18 & 12.57 & 11.62 \\
\hline 22 & 26.90 & 26.24 \\
\hline 26 & 49.90 & 49.58 \\
\hline 30 & 89.49 & 87.60 \\
\hline
\end{tabular}

Table 7: Ten Program Locations

\begin{tabular}{|r|c|c|}
\hline $\mathrm{N}$ & 9 Justice Cond. & 1 Justice Cond. \\
\hline 6 & 0.81 & 0.23 \\
\hline 8 & 4.26 & 3.26 \\
\hline 10 & 10.44 & 9.11 \\
\hline 12 & 21.05 & 18.79 \\
\hline 14 & 38.66 & 35.74 \\
\hline 16 & 64.68 & 60.94 \\
\hline
\end{tabular}

\section{References}

$\left[\mathrm{BCM}^{+} 92\right]$ J. R. Burch, E. M. Clarke, K. L. McMillan, D. L. Dill, and L. J. Hwang. Symbolic model checking: $10^{20}$ states and beyond. Information and Computation, 98(2):142-170, June 1992.

[CE81] E.M. Clarke and E.A. Emerson. Design and synthesis of synchronization skeletons using branching time temporal logic. In Proc. IBM Workshop on Logics of Programs, volume 131 of Lect. Notes in Comp. Sci., pages 52-71. Springer-Verlag, 1981.

[CGH97] E.M. Clarke, O. Grumberg, and K. Hamaguchi. Another look at LTL model checking. Formal Methods in System Design, 10(1), 1997.

[EL86] E. A. Emerson and C. L. Lei. Efficient model-checking in fragments of the propositional modal $\mu$-calculus. In Proc. First IEEE Symp. Logic in Comp. Sci., pages 267-278, 1986.

[EL87] E. A. Emerson and C. Lei. Modalities for model checking: Branching time logic strikes back. Science of Computer Programming, 8:275-306, 1987.

[Fra86] N. Francez. Fairness. Springer-Verlag, 1986.

[GPSS80] D. Gabbay, A. Pnueli, S. Shelah, and J. Stavi. On the temporal analysis of fairness. In Proc. 7th ACM Symp. Princ. of Prog. Lang., pages 163-173, 1980.

[HKSV97] R. H. Hardin, R. P. Kurshan, S. K. Shukla, and M.Y. Vardi. A new heuristic for bad cycle detection using BDDs. In O. Grumberg, editor, O. Grumberg, editor, Proc. Proc. $9^{\text {th }}$ Intl. Conference on Computer Aided Verification, (CAV'97), volume 1254 of Lect. Notes in Comp. Sci., Springer-Verlag, pages 268 - 278, 1997.

[HT96] M.R. Henzinger and J.A. Telle. Faster algorithms for the nonemptiness of street automata and for communication protocol prunning. In Proceedings of the 5th Scandinavian Workshop on Algorithm Theory, pages 10-20, 1996.

[HTKB92] R. Hojati, H. Touati, R.P. Kurshan, and R.K. Brayton. Efficient $\omega$-regular language containment. In In G.V. Bochmann and D.K. Probst, editors, Proc. $4^{\text {th }}$ Intl. Conference on Computer Aided Verification (CAV'92), volume 697 of Lect. Notes in Comp. Sci., Springer-Verlag, number 663 in Lect. Notes in Comp. Sci., pages 396-409. SPringerVerlag, 1992. 
[KP00] Y. Kesten and A. Pnueli. Verification by finitary abstraction. Information and Computation, 163:203-243, 2000.

[KPR98] Y. Kesten, A. Pnueli, and L. Raviv. Algorithmic verification of linear temporal logic specifications. In K.G. Larsen, S. Skyum, and G. Winskel, editors, Proc. 25th Int. Colloq. Aut. Lang. Prog., volume 1443 of Lect. Notes in Comp. Sci., pages 1-16. SpringerVerlag, 1998.

[Kur95] R.P. Kurshan. Computer Aided Verification of Coordinating Processes. Princeton University Press, Princeton, New Jersey, 1995.

[Lic91] O. Lichtenstein. Decidability, Completeness, and Extensions of Linear Time Temporal Logic. PhD thesis, Weizmann Institute of Science, 1991.

[LP85] O. Lichtenstein and A. Pnueli. Checking that finite state concurrent programs satisfy their linear specification. In Proc. 12th ACM Symp. Princ. of Prog. Lang., pages 97-107, 1985 .

[LPS81] D. Lehmann, A. Pnueli, and J. Stavi. Impartiality, justice and fairness: The ethics of concurrent termination. In Proc. 8th Int. Colloq. Aut. Lang. Prog., volume 115 of Lect. Notes in Comp. Sci., pages 264-277. Springer-Verlag, 1981.

[LPZ85] O. Lichtenstein, A. Pnueli, and L. Zuck. The glory of the past. In Proc. Conf. Logics of Programs, volume 193 of Lect. Notes in Comp. Sci., pages 196-218. Springer-Verlag, 1985.

[MP91a] Z. Manna and A. Pnueli. Completing the temporal picture. Theor. Comp. Sci., 83(1):97130, 1991.

[MP91b] Z. Manna and A. Pnueli. The Temporal Logic of Reactive and Concurrent Systems: Specification. Springer-Verlag, New York, 1991.

[MP95] Z. Manna and A. Pnueli. Temporal Verification of Reactive Systems: Safety. SpringerVerlag, New York, 1995.

[PS96] A. Pnueli and E. Shahar. A platform for combining deductive with algorithmic verification. In R. Alur and T. Henzinger, editors, R. Alur and T. Henzinger, editors, Proc. $8^{\text {th }}$ Intl. Conference on Computer Aided Verification (CAV'96), volume 1102 of Lect. Notes in Comp. Sci., Springer-Verlag, pages 184-195, 1996.

[QS82] J.P. Queille and J. Sifakis. Specification and verification of concurrent systems in cesar. In M. Dezani-Ciancaglini and M. Montanari, editors, International Symposium on Programming, volume 137 of Lect. Notes in Comp. Sci., pages 337-351. Springer-Verlag, 1982.

[RBS00] K. Ravi, R. Bloem, and F. Somenzi. A comparative study of symbolic algorithms for the computation of fair cycles. In W. A. Hunt, Jr. and S. D. Johnson, editors, Formal Methods in Computer Aided Design, volume 1954 of Lect. Notes in Comp. Sci., pages 143-160. Springer-Verlag, November 2000.

[SdRG89] F.A. Stomp, W.-P. de Roever, and R.T. Gerth. The $\mu$-calculus as an assertion language for fairness arguments. Inf. and Comp., 82:278-322, 1989. 
[VW86] M.Y. Vardi and P. Wolper. An automata-theoretic approach to automatic program verification. In Proc. First IEEE Symp. Logic in Comp. Sci., pages 332-344, 1986.

[Yan99] Z. Yang. Performance analysis of symbolic reachability algorithms in model checking. Master's thesis, Rice University, 1999. 